OPEN ACCESS

Edited by:

Matthias Sigler,

University of Göttingen, Germany

Reviewed by:

Omar R. J. Tamimi

King Fahd Medical City, Saudi Arabia

Keiichi Hirono,

University of Toyama, Japan

*Correspondence:

Takamichi Ishikawa ishikawa@hama-med.ac.jp

Specialty section

This article was submitted to

Pediatric Cardiology,

a section of the journal

Frontiers in Pediatrics

Received: 30 December 2021 Accepted: 10 February 2022 Published: 03 March 2022

Citation:

Ishikawa T, Uchiyama H, Mogi S and Ohtani H (2022) Case Report: Structural Changes in the Coronary Vessel Wall in a Patient With

Incomplete Kawasaki Disease.

Front. Pediatr. 10:845723

doi: $10.3389 /$ fped.2022.845723

\section{Case Report: Structural Changes in the Coronary Vessel Wall in a Patient With Incomplete Kawasaki Disease}

\author{
Takamichi Ishikawa $^{1 *}$, Hiroki Uchiyama ${ }^{1}$, Satoshi Mogi ${ }^{2}$ and Hayato Ohtani ${ }^{2}$ \\ ${ }^{1}$ Department of Pediatrics, Hamamatsu University School of Medicine, Hamamatsu, Japan, ${ }^{2}$ Division of Cardiology, Internal \\ Medicine 3, Hamamatsu University School of Medicine, Hamamatsu, Japan
}

Background: Kawasaki disease (KD) is an acute systemic vasculitis of infants and young children that affects medium-sized vessels. Conventional cardiac imaging techniques, such as cardiac catheterization, are useful for characterizing the coronary arterial lesion $(C A L)$ size and luminal diameter of the diseased coronary artery segment in patients with KD, but there are limitations to the visualization of the detailed vascular anatomy. Optical coherence tomography (OCT) is a high-resolution intracoronary arterial imaging modality that can distinguish the three layers of the coronary arterial wall. Several studies have reported coronary artery wall abnormalities in KD patients with coronary arterial aneurysm or regressed aneurysm. However, there have been no reports on changes in the coronary artery wall in cases of incomplete KD without CAL.

Case Presentation: We herein report an 11-year-old girl with a history of incomplete $\mathrm{KD}$ without coronary arterial aneurysms. She had been diagnosed with perimembranous ventricular septal defect (VSD) after birth and had experienced incomplete KD at 1 year old. During her hospitalization for KD, she did not receive intravenous immunoglobulin (IVIG), because she did not meet the Harada score or criteria for treatment in patients with incomplete KD established by the American Heart Association. No dilatation or coronary artery aneurysm were observed on transthoracic echocardiography in the acute or follow-up period. At 11 years old, she received cardiac catheterization and coronary angiography (CAG) for the evaluation of a VSD and follow-up of KD. CAG demonstrated no aneurysm, dilatation, or significant stenosis of the coronary arteries. We performed an OCT study, which revealed the presence of intimal thickening, disruption of the media, and neovascularization in the left anterior descending artery.

Conclusion: OCT demonstrates the structural changes of CA even in the patient with incomplete KD who have not been treated with IVIG.

\footnotetext{
Keywords: Kawasaki disease, incomplete Kawasaki disease, optical coherence tomography, coronary arterial lesion, intravenous immunoglobulin
}

\section{INTRODUCTION}

Kawasaki disease (KD) is an acute systemic vasculitis of infants and young children that affects medium-sized vessels. Coronary arterial lesions (CALs) are a serious complication occurring in 5\% of treated and $25-30 \%$ of untreated KD patients $(1,2)$. Conventional cardiac imaging techniques, such as echocardiography, computed tomography, coronary angiography, cardiac magnetic 
resonance imaging, and invasive angiography, are useful for determining the aneurysm size and luminal diameter of the diseased coronary artery segment. However, those modalities are of limited use for the visualization of detailed vascular anatomical data.

Optical coherence tomography (OCT) is a high-resolution intracoronary arterial imaging modality that is able to distinguish the three layers of the coronary arterial wall (3). However, while several studies have used OCT to evaluate CAL in patients with $\mathrm{KD}$ (4-6), there have been no reports of changes in the coronary artery wall in cases of incomplete $\mathrm{KD}$ without CAL.

\section{CASE DESCRIPTION}

An 11-year-old Japanese girl was admitted to undergo cardiac catheterization and coronary angiography (CAG) for the evaluation of a ventricular septal defect (VSD) and follow-up of KD.

\section{Past IIIness}

A heart murmur had been detected after birth, and she had been diagnosed with perimembranous VSD by echocardiography. She had been followed-up for VSD at the outpatient unit periodically.
At 17 months old, she was referred by an affiliated clinic for a fever for the past 2 days, polymorphous rash, and erythema of the hand. On admission, laboratory test findings were as follows: white blood cell counts $8,000 / \mathrm{mm}^{3}$, hemoglobin $14.4 \mathrm{~g} / \mathrm{dL}$, hematocrit $41.0 \%$, platelet count $20.3 \times 10^{4} / \mathrm{mm}^{3}$, serum albumin $4.4 \mathrm{~g} / \mathrm{dL}$, alanine transaminase $38 \mathrm{IU} / \mathrm{L}$, and Creactive protein (CRP) $8.59 \mathrm{mg} / \mathrm{dL}$. Blood culture and serology of Epstein Barr virus to rule out the infectious origin of symptoms were negative. She was diagnosed with incomplete KD. Oral treatment with medium-dose acetylsalicylic acid (30 $\mathrm{mg} / \mathrm{kg} /$ day) immediately improved these symptoms. Four days after admission, the CRP level decreased to $1.25 \mathrm{mg} / \mathrm{dL}$. She was not on intravenous immunoglobulin (IVIG), because her Harada score was $<4 / 7(2 / 7)(7)$, and she did not meet the criteria for incomplete $\mathrm{KD}$ treatment established by the American Heart Association (1).

After the fever had disappeared, low-dose aspirin therapy $(5 \mathrm{mg} / \mathrm{kg} /$ day) was started and then completed 2 months later. No dilatation or coronary artery aneurysm was observed on transthoracic echocardiography in the acute or follow-up period. There were no obvious coronary aneurysms on the cardiac catheterization to evaluate the VSD 3 months after the onset of KD (Figure 1). She was followed up for VSD without surgery, as her pulmonary blood flow/systemic blood flow ratio (Qp/Qs) was 1.3.

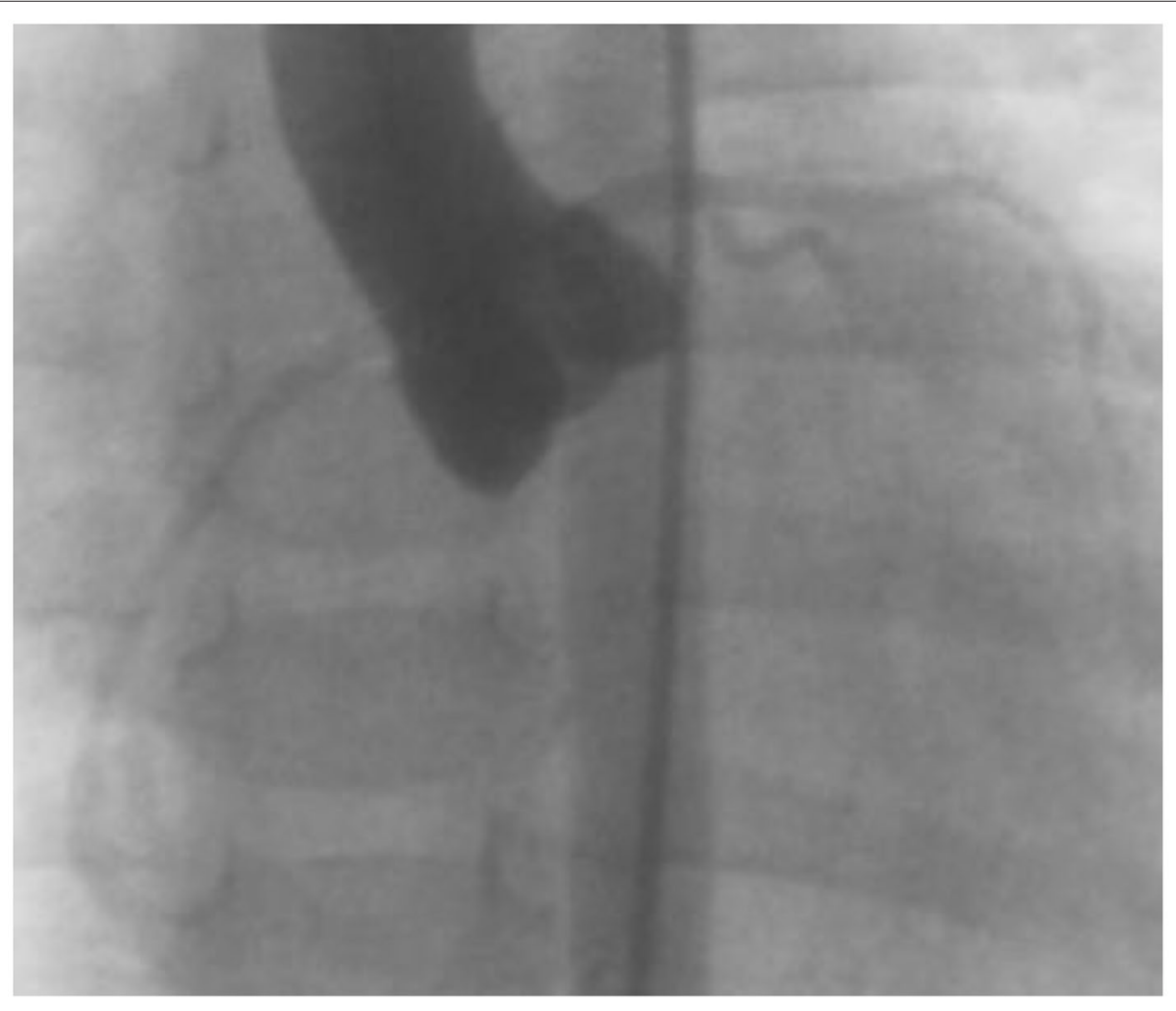

FIGURE 1 | An aortic angiogram showing no coronary artery aneurysm. 


\section{Findings on Admission and Management}

Her height and weight were $139.9 \mathrm{~cm}(-0.5$ standard deviations $[\mathrm{SD}])$ and $33.8 \mathrm{~kg}(-0.4 \mathrm{SD})$, respectively. Her heart rate was $80 \mathrm{bpm}$, and blood pressure was $96 / 60 \mathrm{mmHg}$. A physical examination revealed a grade $4 / 6$ pansystolic murmur at the left sternal border in the third to the fourth intercostal space. The liver was not palpable below the right costal margin. There were no abnormal findings in the abdomen or extremities or irregular and blood chemistry test results.

The cardiothoracic ratio on chest X-ray was $52 \%$. The 12 lead electrocardiogram (ECG) revealed mild left ventricular hypertrophy and normal sinus rhythm without ST-T segment change or an abnormal Q wave. Exercise stress testing showed no abnormal findings on ECG and the patient had no symptoms at maximum effort. Two-dimensional transthoracic echocardiography showed a 5.0-mm perimembranous VSD with left-to-right shunt and a normal left ventricular ejection fraction of $70 \%$ without local asynergy.

CAG demonstrated no aneurysm, dilatation, or significant stenosis of the coronary arteries. We performed an OCT study (ILUMIEN ${ }^{\mathrm{TM}}$ OCT Imaging System: St. Jude/LightLab,
St. Paul, MN, USA), which revealed the presence of intimal thickening, disruption of the media, and neovascularization in the left anterior descending artery (Figure 2). There were no abnormal OCT findings in the other coronary artery branches.

CAG and OCT were approved by the Ethics Committee of Hamamatsu University School of Medicine (18029), and the parents provided their informed consent. The patient received surgical closure of the VSD, as the $\mathrm{Qp} / \mathrm{Qs}$ ratio was 1.5 on cardiac catheterization. No coronary artery abnormalities were observed in the perioperative period.

\section{DISCUSSION}

This patient with incomplete $\mathrm{KD}$ had intimal thickening, disruption of the media, and neovascularization in the coronary artery detected by OCT. KD is characterized by severe systemic vasculitis and an acute febrile illness in children. This inflammatory process has been shown to persist for many years after the initial diagnosis, with the proliferation

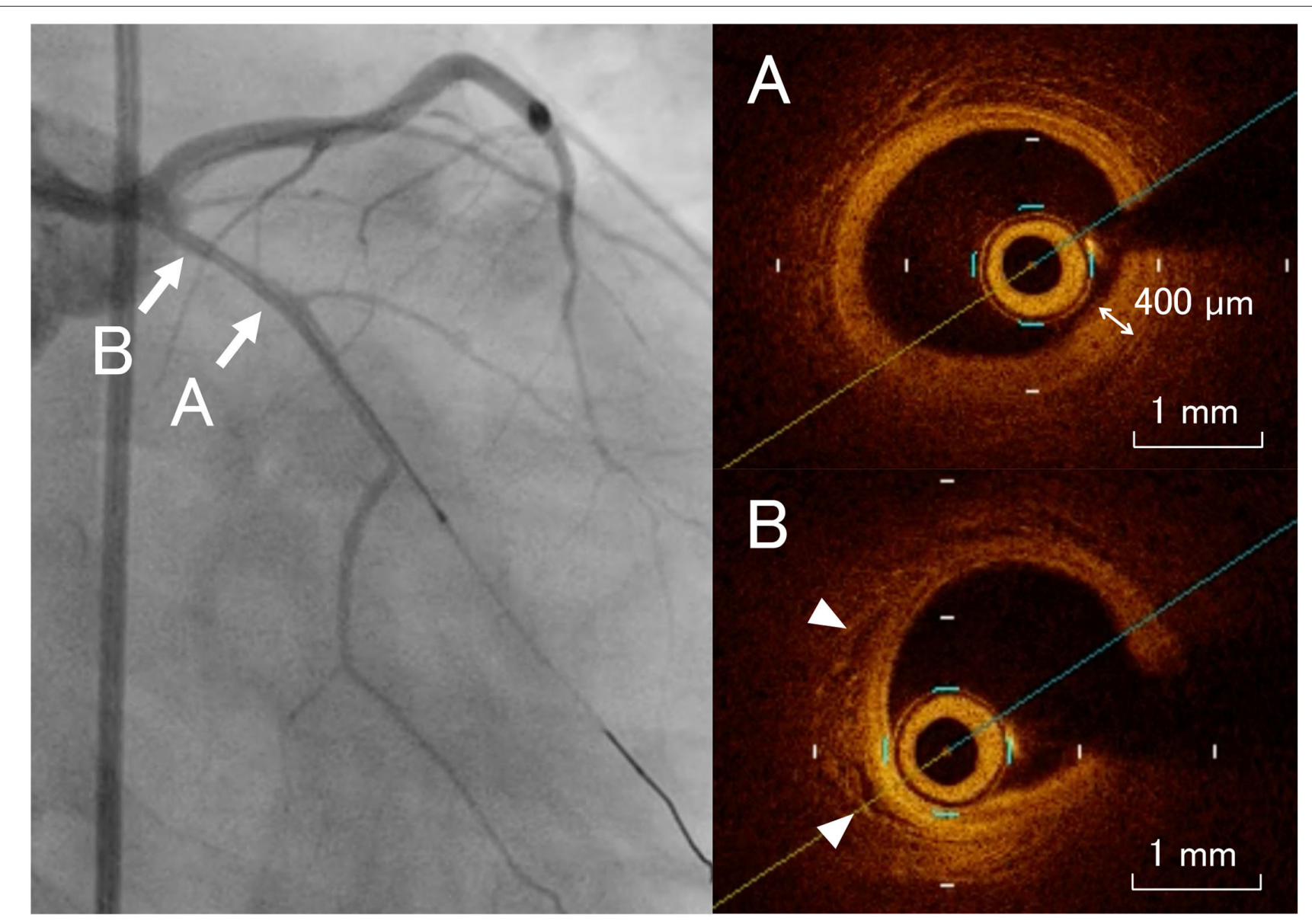

FIGURE 2 | Coronary angiogram images with the corresponding optical coherence tomography examinations. The left anterior descending artery shows intimal thickening, disruption of the media (A), and neovascularization [(B) arrowheads]. 
of dense smooth muscle cells and accumulation of fibrous tissue, leading to intimal thickening (8). Progressive intimal hyperplasia is associated with hypoxia of the cells, which further promotes angiogenesis through hypoxia-inducible transcription factors that then induce the transcription of proangiogenic genes (9). These findings suggest the importance of the precise evaluation of coronary wall structural changes in at-risk KD patients.

OCT is a high-resolution intracoronary arterial imaging modality that is able to distinguish the three layers of the coronary arterial wall: the intima, media, and adventitia (3). Several studies have used OCT to evaluate CAL in patients with KD (4-6). A previous study reported that calcification and destruction of the media layer, neovascularization, and white thrombi were found in not only segments with aneurysm, but also in those with regressed aneurysm (6). However, there have been no previous reports of changes in the coronary artery wall in cases of incomplete $\mathrm{KD}$ without CAL.

The present patient did not receive IVIG therapy, as she did not meet the Harada score cut-off (7) or the criteria for the treatment of patients with incomplete $\mathrm{KD}$ established by the American Heart Association (1). In cases with incomplete $\mathrm{KD}$, the timely commencement of IVIG therapy is often hampered by the lack of definitive diagnostic symptoms. As a result, some incomplete $\mathrm{KD}$ patients experience spontaneous defervescence before IVIG administration as in the present case. However, it has been suggested that there is persistent mild systemic inflammation in incomplete $\mathrm{KD}$ patients who experience spontaneous defervescence without receiving IVIG (10). Patients with KD who are not treated with IVIG therapy have a $25-30 \%$ risk of developing CALs $(1,2)$. Other studies have shown that the CAL complication rate within 30 days of illness in the $\mathrm{KD}$ patients received aspirin alone was $20-40 \%(1,11)$. In the previous reports, all $\mathrm{KD}$ patients with abnormal findings on OCT were diagnosed with complete $\mathrm{KD}$ and/or received delayed administration of IVIG or showed IVIG resistance (4-6). Prompt IVIG therapy is the currently internationally recognized method of managing $\mathrm{KD}$ with the specific aim of preventing CALs (1). However, these findings suggest that it is possible to prevent not only CALs but also structural changes of the coronary vessel wall if IVIG is administrated without delay.

The present case had abnormalities of the coronary artery wall, despite a lack of CALs. While the cause and pathogenesis of these findings were unclear, previous studies have shown that intimal thickening, vascular endothelial dysfunction, and impaired coronary microcirculation are observed, even in patients with apparently normal coronary arteries $(12,13)$. Coronary artery remodeling and neoangiogenesis may continue for a decade after the development of acute KD (8). Previous studies of coronary artery specimens from KD patients have demonstrated the phenomenon of vascular senescence, which is similar to atherosclerosis in adults $(14,15)$. The use of OCT to identify atherosclerotic plaque and detect calcification in adults with coronary artery disease has been well described.
The correlations between the pathological and OCT findings have described in this population (16). Because this procedure was introduced in this field very recently, the number of reports that have investigated the coronary arteries of $\mathrm{KD}$ patients by OCT has been limited. However, a recent report using OCT demonstrated that after KD $5-40 \%$ of patients had intimal hyperplasia, fibrosis, and cellular infiltration in segments with no history of aneurysm or coronary dilatation (6). These findings suggest that OCT can detect structural abnormalities in the coronary vessel wall that are missed by conventional imaging.

The findings in the present and past reports suggest that we should be aware of not only CALs but also structural changes of the coronary vessel wall when we encounter patients who have not been treated with IVIG or for whom the administration of IVIG is delayed or patients who show IVIG resistance. Vascular structural abnormalities lead to vascular dysfunction and impaired blood flow. Endothelial dysfunction, which is caused by endothelial damage, is an early feature of atherosclerosis $(17,18)$. Therefore, we believe that these patients may require careful follow-up with monitoring to detect the future development of arteriosclerosis. In addition, the prevention of additional cardiovascular risk factors, such as obesity, hypertension, hyperlipidemia, and smoking may be required in such patients. Further large-scale and prospective studies to evaluate the vascular structure and the association with an increased risk of late adverse events in these patients are needed. The accumulation of evidence using OCT may contribute to risk stratification and the construction of a management system for KD patients.

\section{DATA AVAILABILITY STATEMENT}

The raw data supporting the conclusions of this article will be made available by the authors, without undue reservation.

\section{ETHICS STATEMENT}

The studies involving human participants were reviewed and approved by the Ethics Committee of Hamamatsu University School of Medicine. Written informed consent to participate in this study was provided by the participants' legal guardian/next of kin.

\section{AUTHOR CONTRIBUTIONS}

TI wrote the manuscript. All authors listed have made a substantial, direct, and intellectual contribution to the work and approved it for publication. All authors contributed to the article and approved the submitted version.

\section{FUNDING}

This work was supported by JSPS KAKENHI Grant Number JP18K07787. 


\section{REFERENCES}

1. Newburger JW, Takahashi M, Gerber MA, Gewitz MH, Tani LY, Burns JC, et al. Diagnosis, treatment, and long-term management of Kawasaki disease: a statement for health professionals from the Committee on Rheumatic Fever, Endocarditis and Kawasaki Disease, Council on Cardiovascular Disease in the Young, American Heart Association. Circulation. (2004) 110:274771. doi: 10.1161/01.CIR.0000145143.19711.78

2. Senzaki H. Long-term outcome of Kawasaki disease. Circulation. (2008) 118:2763-72. doi: 10.1161/CIRCULATIONAHA.107.749515

3. Akasaka T, Kubo T, Mizukoshi M, Tanaka A, Kitabata H, Tanimoto T, et al. Pathophysiology of acute coronary syndrome assessed by optical coherence tomography. J Cardiol. (2010) 56:8-14. doi: 10.1016/j.jjcc.2010.05.005

4. Kakimoto N, Suzuki H, Kubo T, Suenaga T, Takeuchi T, Shibuta S, et al. Evaluation of coronary arterial lesions due to Kawasaki disease using optical coherence tomography. Can J Cardiol. (2014) 30:956.e79. doi: 10.1016/j.cjca.2014.04.028

5. Dionne A, Ibrahim R, Gebhard C, Bakloul M, Selly JB, Leye M, et al. Coronary Wall Structural Changes in Patients With Kawasaki Disease: New Insights From Optical Coherence Tomography (OCT). J Am Heart Assoc. (2015) 4:e001939. doi: 10.1161/JAHA.115.001939

6. Dionne A, Ibrahim R, Gebhard C, Benovoy M, Leye M, Déry J, et al. Difference between persistent aneurysm, regressed aneurysm, and coronary dilation in Kawasaki Disease: an optical coherence tomography study. Can J Cardiol. (2018) 34:1120-8. doi: 10.1016/j.cjca.2018.05.021

7. Harada K. Intravenous gamma-globulin treatment in Kawasaki disease. Acta Paediatr Jpn. (1991) 33:805-10. doi: 10.1111/j.1442-200X.1991.tb02612.x

8. Suzuki A, Miyagawa-Tomita S, Komatsu K, Nishikawa T, Sakomura Y, Horie $\mathrm{T}$, et al. Active remodeling of the coronary arterial lesions in the late phase of Kawasaki disease: immunohistochemical study. Circulation. (2000) 101:293541. doi: 10.1161/01.CIR.101.25.2935

9. Freeman AF, Crawford SE, Cornwall ML, Garcia FL, Shulman ST, Rowley AH. Angiogenesis in fatal acute Kawasaki disease coronary artery and myocardium. Pediatr Cardiol. (2005) 26:578-84. doi: 10.1007/s00246-005-0801-2

10. Takahashi T, Sakakibara H, Morikawa Y, Miura M. Development of coronary artery lesions in indolent Kawasaki disease following initial spontaneous defervescence: a retrospective cohort study. Pediatr Rheumatol Online J. (2015) 13:44. doi: 10.1186/s12969-015-0042-8

11. Oates-Whitehead RM, Baumer JH, Haines L, Love S, Maconochie IK, Gupta A, et al. Intravenous immunoglobulin for the treatment of Kawasaki disease in children. Cochrane Database Syst Rev. (2003) 2003:Cd004000. doi: 10.1002/14651858.CD004000
12. Takahashi K, Oharaseki T, Naoe S. Pathological study of postcoronary arteritis in adolescents and young adults: with reference to the relationship between sequelae of Kawasaki disease and atherosclerosis. Pediatr Cardiol. (2001) 22:138-42. doi: 10.1007/s002460010180

13. Niboshi A, Hamaoka K, Sakata K, Yamaguchi N. Endothelial dysfunction in adult patients with a history of Kawasaki disease. Eur J Pediatr. (2008) 167:189-96. doi: 10.1007/s00431-007-0452-9

14. Cheung YF, Yung TC, Tam SC, Ho MH, Chau AK. Novel and traditional cardiovascular risk factors in children after Kawasaki disease: implications for premature atherosclerosis. J Am Coll Cardiol. (2004) 43:1204. doi: 10.1016/j.jacc.2003.08.030

15. Fukazawa R, Ikegam E, Watanabe M, Hajikano M, Kamisago M, Katsube Y, et al. Coronary artery aneurysm induced by Kawasaki disease in children show features typical senescence. Circ J. (2007) 71:709-15. doi: 10.1253/circj.71.709

16. Tearney GJ, Regar E, Akasaka T, Adriaenssens T, Barlis P, Bezerra HG, et al. Consensus standards for acquisition, measurement, and reporting of intravascular optical coherence tomography studies: a report from the International Working Group for Intravascular Optical Coherence Tomography Standardization and Validation. J Am Coll Cardiol. (2012) 59:1058-72. doi: 10.1016/j.jacc.2011.09.079

17. Bürrig KF. The endothelium of advanced arteriosclerotic plaques in humans. Arterioscler Thromb. (1991) 11:1678-89. doi: 10.1161/01.ATV.11.6.1678

18. Ross R. Atherosclerosis-an inflammatory disease. N Engl J Med. (1999) 340:115-26. doi: 10.1056/NEJM199901143400207

Conflict of Interest: The authors declare that the research was conducted in the absence of any commercial or financial relationships that could be construed as a potential conflict of interest.

Publisher's Note: All claims expressed in this article are solely those of the authors and do not necessarily represent those of their affiliated organizations, or those of the publisher, the editors and the reviewers. Any product that may be evaluated in this article, or claim that may be made by its manufacturer, is not guaranteed or endorsed by the publisher.

Copyright (c) 2022 Ishikawa, Uchiyama, Mogi and Ohtani. This is an open-access article distributed under the terms of the Creative Commons Attribution License (CC $B Y)$. The use, distribution or reproduction in other forums is permitted, provided the original author(s) and the copyright owner(s) are credited and that the original publication in this journal is cited, in accordance with accepted academic practice. No use, distribution or reproduction is permitted which does not comply with these terms. 